\title{
Neimark-Sacker Bifurcation of a Third Order Difference Equation
}

\author{
Asmaa Shareef ${ }^{1}$ and Marwan Aloqeili ${ }^{1 *}$ \\ ${ }^{1}$ Department of Mathematics, Faculty of Science, Birzeit University, Birzeit, Palestine \\ ${ }^{*}$ Corresponding author E-mail: maloqeili@birzeit.edu
}

\section{Article Info}

Keywords: Equilibrium, Difference equation, Neimark-Sacker Bifurcation, Stability.

2010 AMS: $39 A 28$

Received: 15 February 2019

Accepted: 25 April 2019

Available online: 17 June 2019

\begin{abstract}
In this paper, we investigate the bifurcation of a third order rational difference equation. Firstly, we show that the equation undergoes a Neimark-Sacker bifurcation when the parameter reaches a critical value. Then, we consider the direction of the Neimark-Sacker bifurcation. Finally, we give some numerical simulations of our results.
\end{abstract}

\section{Introduction}

Bifurcation is an important dynamic behavior of some dynamical systems. Some difference equations exhibits different kinds of bifurcation including period-doubling bifurcation, saddle-node bifurcation and Neimark-Sacker bifurcation. In this paper, we show that a third order rational difference equation exhibits Neimark-Sacker bifurcation. This type of bifurcation exits when the Jacobian matrix of a system of difference equations has complex eigenvalues of modulus one. In [1], the author studied the dynamics of the third order difference equation

$$
x_{n+1}=\frac{\beta x_{n}+\delta x_{n-2}}{A+B x_{n}+C x_{n-1}}
$$

Using appropriate change of variables, equation (1.1) becomes

$$
x_{n+1}=\frac{\beta x_{n}+x_{n-2}}{A+B x_{n}+x_{n-1}}
$$

where $A \geq 0, \beta, B>0$. The author gives dynamic properties of solutions of this equation. In [2], the authors considered the difference equation

$$
x_{n+1}=\frac{\beta x_{n}+\alpha x_{n-2}}{1+x_{n-1}}
$$

They show that this equation undergoes a Neimark-Sacker bifurcation and give the direction of the bifurcation. In this paper, we consider the third order rational difference equation

$$
x_{n+1}=\frac{\beta x_{n}+x_{n-2}}{A+x_{n-1}}
$$

where $A \in(0,1), \beta>0$ and nonnegative initial conditions $x_{-2}, x_{-1}$ and $x_{0}$. Firstly, we show that the unique positive equilibrium $X^{*}=$ $\beta-A+1$ is locally asymptotically stable if $\beta>(1-A) /(1+A)$. Then, we show that equation (1.2) undergoes a Neimark-Sacker bifurcation by converting this equation to a first order system and showing that the Jacobian matrix of the linearized system has a pair of complex

Email addresses and ORCID numbers: asma_aqel@yahoo.com, https://orcid.org/0000-0001-8109-5811 (A. Shareef), https://orcid.org/0000-0002-9030-1717 (M. Aloqeili),maloqeili@birzeit.edu 
conjugated eigenvalues of modulus one and a real eigenvalue in the interval $(0,1)$. Equation (1.2) is a special case of the following one which was considered in [3]

$$
x_{n+1}=\frac{\alpha+\beta x_{n}+\gamma x_{n-1}+x_{n-2}}{A+x_{n-1}}
$$

setting $\alpha=\gamma=0$, we get equation (1.2). The authors in [3] proved that every solution of equation (1.3) is bounded.

The rest of the article is organized as follows: in section 2, we give condition for local asymptotic stability. Then, we show in section 3 that equation (1.2) undergoes a Neimark-Sacker bifurcation. In section 4, the direction of bifurcation is considered. Finally, some numerical simulations are given.

\section{Local stability}

In this section, we study local stability of the unique positive equilibrium of equation (1.2). We apply Jury's test to the characteristic polynomial of the linearized equation. Jury's conditions provide an algebraic test that determines whether the roots of a polynomial lie within the unit circle. Jury's conditions consist of a test for necessary conditions and a test for sufficient conditions. For a polynomial of the form:

$$
f(z)=a_{n} z^{n}+a_{n-1} z^{n-1}+\cdots+a_{1} z+a_{0}
$$

The necessary conditions for stability are: $f(1)>0$ and $(-1)^{n} f(-1)>0$, while the sufficient conditions for stability are given by:

$$
\left|a_{0}\right|<a_{n}, \quad\left|b_{0}\right|>\left|b_{n-1}\right|, \quad\left|c_{0}\right|>\left|c_{n-2}\right|, \cdots
$$

where $b_{k}=\left|\begin{array}{cc}a_{0} & a_{n-k} \\ a_{n} & a_{k}\end{array}\right|, \quad c_{k}=\left|\begin{array}{cc}b_{0} & b_{n-1-k} \\ b_{n-1} & b_{k}\end{array}\right|$

We need the following theorem

Theorem 2.1. (Viète Theorem [4]) Consider the following polynomial of degree $n$

$$
f(z)=a_{n} z^{n}+a_{n-1} z^{n-1}+\cdots+a_{1} z+a_{0}
$$

Then, the $n$ roots of $f$ (counting multiplicities) $z_{1}, z_{2}, \cdots, z_{n}$ satisfy the following relations

$$
\begin{gathered}
z_{1}+z_{2}+\cdots+z_{n-1}+z_{n}=\frac{-a_{n-1}}{a_{n}} \\
\left(z_{1} z_{2}+z_{1} z_{3}+\cdots+z_{1} z_{n}\right)+\left(z_{2} z_{3}+z_{2} z_{4}+\cdots+z_{2} z_{n}\right)+\cdots+z_{n-1} z_{n}=\frac{a_{n-2}}{a_{n}} \\
\vdots \\
z_{1} z_{2} \cdots z_{n}=(-1)^{n} \frac{a_{0}}{a_{n}}
\end{gathered}
$$

Firstly, we convert the third order equation (1.2) to the first order system

$$
\begin{aligned}
x_{n+1} & =\frac{\beta x_{n}+z_{n}}{A+y_{n}} \\
y_{n+1} & =x_{n} \\
z_{n+1} & =y_{n}
\end{aligned}
$$

The system has two fixed points, the first one is the zero fixed point $(0,0,0)$ and a positive fixed point

$$
X^{*}=(\beta-A+1, \beta-A+1, \beta-A+1), \quad \beta+1>A
$$

Viète's theorem will be used to show that the Jacobian matrix of the above system has a pair of complex eigenvalues of modulus one. The following theorem gives a condition for local stability of $X^{*}$. Let

$$
\beta^{*}=\frac{1-A}{1+A}
$$

Theorem 2.2. The positive fixed point is stable if $\beta>\beta^{*}$ and unstable if $\beta<\beta^{*}$

Proof. The Jacobian matrix of the system is

$$
J=\left(\begin{array}{ccc}
\frac{\beta}{A+y_{n}} & \frac{-\left(\beta x_{n}+z_{n}\right)}{\left(A+y_{n}\right)^{2}} & \frac{1}{A+y_{n}} \\
1 & 0 & 0 \\
0 & 1 & 0
\end{array}\right)
$$

At the positive fixed point

$$
J=\left(\begin{array}{ccc}
\frac{\beta}{\beta+1} & \frac{-(\beta-A+1)}{\beta+1} & \frac{1}{\beta+1} \\
1 & 0 & 0 \\
0 & 1 & 0
\end{array}\right)
$$


The characteristic equation of the Jacobian matrix $J$ is

$$
p(\lambda)=|\lambda I-J|=\lambda^{3}-\frac{\beta}{\beta+1} \lambda^{2}+\frac{\beta-A+1}{\beta+1} \lambda-\frac{1}{\beta+1}
$$

To study the stability of $X^{*}$ we use Jury's conditions

$$
\begin{gathered}
p(1)=\frac{\beta-A+1}{\beta+1}>0 \\
(-1)^{3} p(-1)=2+\frac{\beta-A+1}{\beta+1}>0
\end{gathered}
$$

The sufficient conditions are, $\left|a_{0}\right|<a_{3}$ and $\left|b_{0}\right|>\left|b_{2}\right|$ where

$$
a_{0}=\frac{-1}{\beta+1}, \quad a_{1}=\frac{\beta-A+1}{\beta+1}, \quad a_{2}=\frac{-\beta}{\beta+1}, \quad a_{3}=1
$$

and

$$
b_{0}=\left|\begin{array}{ll}
a_{0} & a_{3} \\
a_{3} & a_{0}
\end{array}\right|, \quad b_{2}=\left|\begin{array}{ll}
a_{0} & a_{1} \\
a_{3} & a_{2}
\end{array}\right|
$$

The condition $\left|a_{0}\right|<a_{3}$ is trivially satisfied. Now,

$$
b_{0}=\frac{1}{(\beta+1)^{2}}-1, \quad \text { thus } \quad\left|b_{0}\right|=1-\frac{1}{(\beta+1)^{2}}=\frac{\beta^{2}+2 \beta}{(\beta+1)^{2}}
$$

and

$$
b_{2}=\left|\begin{array}{cc}
\frac{-1}{\beta+1} & \frac{\beta-A+1}{\beta+1} \\
1 & \frac{-\beta}{\beta+1}
\end{array}\right|=\frac{-\beta^{2}-\beta-1+A \beta+A}{(\beta+1)^{2}}
$$

We consider two cases. The first case is

$$
\frac{-\beta^{2}-\beta-1+A \beta+A}{(\beta+1)^{2}}>0
$$

then $\left|b_{2}\right|=\frac{-\beta^{2}-\beta-1+A \beta+A}{(\beta+1)^{2}}$. The condition $\left|b_{0}\right|>\left|b_{2}\right|$ is satisfied if and only if

$$
\frac{\beta^{2}+2 \beta}{(\beta+1)^{2}}>\frac{-\beta^{2}-\beta-1+A \beta+A}{(\beta+1)^{2}}
$$

which is equivalent to

$$
\frac{2 \beta+1-A}{\beta+1}>0
$$

the last inequality is satisfied since $\beta-A+1>0$. The second case is when

$$
\frac{-\beta^{2}-\beta-1+A \beta+A}{(\beta+1)^{2}}<0
$$

So

$$
\left|b_{2}\right|=\frac{\beta^{2}+\beta+1-A \beta-A}{(\beta+1)^{2}}
$$

Now, $\left|b_{0}\right|>\left|b_{2}\right|$ if

$$
\frac{\beta^{2}+2 \beta}{(\beta+1)^{2}}>\frac{\beta^{2}+\beta+1-A \beta-A}{(\beta+1)^{2}}
$$

which is satisfied if and only if

$$
\beta>\frac{1-A}{1+A}
$$

The proof is complete. 


\section{Existence of Neimark-Sacker bifurcation}

In this section, we show that equation (1.2) undergoes a Neimark-Sacker bifurcation by proving the existence of a pair of complex conjugate eigenvalues of modulus one.

Theorem 3.1. When $\beta=\beta^{*}=\frac{1-A}{1+A}$, polynomial (2.1) has two complex conjugate roots of modulus one and another real root that lies inside the unit circle. Moreover for $A \in(0,1)$ the Neimark Sacker bifurcation conditions are satisfied.

The theorem will be proved through the following lemmas

Lemma 3.1. The characteristic polynomial (2.1) has two complex roots, $\lambda_{1}, \lambda_{2}=\bar{\lambda}_{1}$ and a real root $\lambda_{3}$ in the interval $(0,1)$.

Proof. The derivative of $p(\lambda)$ is given by

$$
p^{\prime}(\lambda)=3 \lambda^{2}-\frac{2 \beta}{\beta+1} \lambda+\frac{\beta-A+1}{\beta+1}
$$

If the discriminant of $p^{\prime}(\lambda)$ is negative then $p(\lambda)$ has complex roots,

$$
\Delta p^{\prime}(\lambda)=\frac{-8 \beta^{2}+12(\beta A+A-1)-24 \beta}{(\beta+1)^{2}}
$$

Using the condition $\beta(A+1)+A-1=0$, we find that

$$
\Delta p^{\prime}(\lambda)=\frac{-8 \beta^{2}-36 \beta}{(\beta+1)^{2}}<0
$$

So $p^{\prime}(\lambda)$ has complex roots. Hence, $p(\lambda)$ has complex roots as well. Since $p(0)=\frac{-1}{\beta+1}<0$ and $p(1)>0$, then there exists $\lambda_{3} \in(0,1)$ such that $p\left(\lambda_{3}\right)=0$, this is the unique real root inside the unit circle.

Lemma 3.2. The complex roots of polynomial (2.1) have modulus one when $\beta=\beta^{*}$. Moreover, the real root $\lambda_{3}=\frac{1}{1+\beta}$.

Proof. Suppose that $\lambda_{1}, \lambda_{2}, \lambda_{3}$ are the roots of $p(\lambda)$ where $\lambda_{2}=\bar{\lambda}_{1}$ and $\lambda_{3}=r_{0}$. We apply Viète theorem to the polynomial $p(\lambda)$. If $\left|\lambda_{1}\right|=\left|\lambda_{2}\right|=1$ and $\lambda_{3}=r_{0}$ then

$$
\begin{gathered}
\lambda_{1}+\lambda_{2}+\lambda_{3}=\frac{-a_{2}}{a_{3}}=\frac{\beta}{\beta+1} \\
\lambda_{1} \lambda_{2}+\lambda_{1} \lambda_{3}+\lambda_{2} \lambda_{3}=\frac{a_{1}}{a_{3}}=\frac{\beta-A+1}{\beta+1} \\
\lambda_{1} \lambda_{2} \lambda_{3}=\frac{-a_{0}}{a_{3}}=\frac{1}{\beta+1}
\end{gathered}
$$

It follows that

$$
\lambda_{1} \lambda_{2} \lambda_{3}=\lambda_{3}=\frac{1}{\beta+1}
$$

Plugging this value of $\lambda_{3}$ into (3.2) and using the fact that $\lambda_{1} \lambda_{2}=1$, we find

$$
\lambda_{1}+\lambda_{2}=-A
$$

Then substitute for $\lambda_{3}$ in (3.1) to get

$$
\lambda_{1}+\lambda_{2}=\frac{\beta}{\beta+1}-\frac{1}{\beta+1}=\frac{\beta-1}{\beta+1}
$$

Therefore,

$$
\lambda_{1}+\lambda_{2}=\frac{\beta-1}{\beta+1}=-A
$$

which implies that

$$
\beta=\frac{1-A}{1+A}
$$

It follows, from the above argument, that there exist a conjugate pair of complex roots on the unit circle.

The roots of the characteristic polynomial depend on the parameters $A$ and $\beta$. Hence, at $\beta^{*}=(1-A) /(1+A)$, these roots are functions of $A$, and will be denoted by $\lambda_{1}^{*}(A)=\lambda_{1}\left(A, \beta^{*}(A)\right), \lambda_{2}^{*}(A)=\lambda_{2}\left(A, \beta^{*}(A)\right)$ and $\lambda_{3}^{*}=\lambda_{3}\left(A, \beta^{*}(A)\right)$ 
Lemma 3.3. The complex roots of polynomial (2.1) are $\lambda_{1,2}^{*}(A)=\exp \pm i \theta^{*}$ where

$$
\theta^{*}=\arccos \left(\frac{-A}{2}\right)
$$

Proof. Let $e^{i \theta}, e^{-i \theta}$ be the roots of $p(\lambda)$, then

$$
\begin{gathered}
e^{3 i \theta}-\frac{\beta}{\beta+1} e^{2 i \theta}+\frac{\beta-A+1}{\beta+1} e^{i \theta}-\frac{1}{\beta+1}=0 \\
\cos 3 \theta+i \sin 3 \theta-\frac{\beta}{\beta+1}(\cos 2 \theta+i \sin 2 \theta)+\frac{\beta-A+1}{\beta+1}(\cos \theta+i \sin \theta)-\frac{1}{\beta+1}=0
\end{gathered}
$$

Separation of real and imaginary parts gives

$$
\cos 3 \theta-\frac{\beta}{\beta+1} \cos 2 \theta=-\frac{\beta-A+1}{\beta+1} \cos \theta+\frac{1}{\beta+1}
$$

and

$$
\sin 3 \theta-\frac{\beta}{\beta+1} \sin 2 \theta=-\frac{\beta-A+1}{\beta+1} \sin \theta
$$

Square both sides of previous equations and add them up, we find that

$$
\begin{gathered}
\cos ^{2} 3 \theta+\sin ^{2} 3 \theta+\left(\frac{\beta}{\beta+1}\right)^{2}\left(\cos ^{2} 2 \theta+\sin ^{2} 2 \theta\right)-\frac{2 \beta}{\beta+1}(\cos 2 \theta \cos 3 \theta+\sin 2 \theta \sin 3 \theta) \\
=\left(\frac{1}{\beta+1}\right)^{2}+\left(\frac{\beta-A+1}{\beta+1}\right)^{2}\left(\cos ^{2} \theta+\sin ^{2} \theta\right)-\frac{2(\beta-A+1)}{(\beta+1)^{2}} \cos \theta
\end{gathered}
$$

It follows that

$$
1+\left(\frac{\beta}{\beta+1}\right)^{2}-\left(\frac{1}{\beta+1}\right)^{2}-\left(\frac{\beta-A+1}{\beta+1}\right)^{2}=\left(\frac{2 \beta}{\beta+1}-\frac{2(\beta-A+1)}{(\beta+1)^{2}}\right) \cos \theta
$$

Simplifying we get

$$
\cos \theta=\frac{(\beta+A-1)}{2 \beta}
$$

Then, evaluating at $\beta=\beta^{*}=\frac{1-A}{1+A}$

$$
\cos \theta=\frac{-A}{2}
$$

Hence for $A \in(0,1), \quad \frac{-1}{2}<\cos \theta<0$. Therefore, there exists $\theta^{*} \in\left(\frac{\pi}{2}, \frac{2 \pi}{3}\right)$ such that

$$
\theta^{*}=\arccos \left(\frac{-A}{2}\right)
$$

Moreover, $\theta^{*} \neq 0, \pm \frac{\pi}{2}, \pm \frac{2 \pi}{3}, \pm \pi$. Consequently, $e^{i k \theta^{*}} \neq 1$ for $k \in\{1,2,3,4\}$.

Lemma 3.4. The condition $\left.\frac{d|\lambda|^{2}}{d \beta}\right|_{\beta=\beta^{*}} \neq 0$ is fulfilled at $\beta=\beta^{*}$.

Proof. Note that

$$
\begin{gathered}
p(\lambda)=\lambda^{3}-\frac{\beta}{\beta+1} \lambda^{2}+\frac{\beta-A+1}{\beta+1} \lambda-\frac{1}{\beta+1} \\
\left.\frac{d|\lambda|^{2}}{d \beta}\right|_{\beta=\beta^{*}}=\frac{d(\lambda \bar{\lambda})}{d \beta}=\lambda \frac{\partial \bar{\lambda}}{\partial \beta}+\bar{\lambda} \frac{\partial \lambda}{\partial \beta} \\
\frac{d|\lambda|^{2}}{d \beta}=\lambda\left(\frac{\partial p(\bar{\lambda})}{\partial \beta} \frac{\partial \bar{\lambda}}{\partial p(\bar{\lambda})}\right)+\bar{\lambda}\left(\frac{\partial p(\lambda)}{\partial \beta} \frac{\partial \lambda}{\partial p(\lambda)}\right) \\
=\lambda\left(\frac{-\bar{\lambda}^{2}+A \bar{\lambda}+1}{(\beta+1)^{2}\left(3 \bar{\lambda}^{2}-\frac{2 \beta}{\beta+1} \bar{\lambda}+\frac{\beta-A+1}{\beta+1}\right.}\right)+\bar{\lambda}\left(\frac{-\lambda^{2}+A \lambda+1}{(\beta+1)^{2}\left(3 \lambda^{2}-\frac{2 \beta}{\beta+1} \lambda+\frac{\beta-A+1}{\beta+1}\right.}\right)
\end{gathered}
$$

After some calculations, the right hand side of the last equation can be written as

$$
\frac{3 A(\beta+1)\left(\bar{\lambda}^{2}+\lambda^{2}\right)-2 \beta A(\lambda+\bar{\lambda})+6 i(\beta+1) \sin \theta\left(\lambda^{2}-\bar{\lambda}^{2}\right)+4 i \beta \sin \theta(\bar{\lambda}-\lambda)+2 A X}{(\beta+1)\left(3(\beta+1) \bar{\lambda}^{2}-2 \beta \bar{\lambda}+X\right)\left(3(\beta+1) \lambda^{2}-2 \beta \lambda+X\right)}
$$


where $X=\beta-A+1$. But

$$
\begin{gathered}
\lambda+\bar{\lambda}=(\cos \theta+i \sin \theta)+(\cos \theta-i \sin \theta)=2 \cos \theta \\
\lambda^{2}+\bar{\lambda}^{2}=(\cos \theta+i \sin \theta)^{2}+(\cos \theta-i \sin \theta)^{2}=2\left(\cos ^{2} \theta-\sin ^{2} \theta\right)=2 \cos (2 \theta) \\
\lambda^{2}-\bar{\lambda}^{2}=(\cos \theta+i \sin \theta)^{2}-(\cos \theta-i \sin \theta)^{2}=4 i \cos \theta \sin \theta=i 2 \sin (2 \theta)
\end{gathered}
$$

Consequently, we have

$$
\frac{d|\lambda|^{2}}{d \beta}=\frac{6 A(\beta+1) \cos (2 \theta)-4 \beta A \cos \theta+(-24(\beta+1) \cos \theta+8 \beta) \sin ^{2} \theta+2 A X}{(\beta+1)\left(3(\beta+1) \bar{\lambda}^{2}-2 \beta \bar{\lambda}+X\right)\left(3(\beta+1) \lambda^{2}-2 \beta \lambda+X\right)}
$$

Now, at $\theta=\theta^{*}, \beta=\beta^{*}$, the last expression becomes

$$
\begin{gathered}
\left.\frac{d|\lambda|^{2}}{d \beta}\right|_{\beta=\beta^{*}}=\frac{8 A \frac{2}{1+A}-2 A^{2}+8 \frac{1-A}{1+A}}{\left(\beta^{*}+1\right)\left(3\left(\beta^{*}+1\right) \bar{\lambda}^{2}-2 \beta \bar{\lambda}+X^{*}\right)\left(3\left(\beta^{*}+1\right) \lambda^{2}-2 \beta^{*} \lambda+X^{*}\right)} \\
=\frac{-2(A-2)(A+2)(A+1)}{(1+A)\left(\beta^{*}+1\right)\left(3\left(\beta^{*}+1\right) \bar{\lambda}^{2}-2 \beta^{*} \bar{\lambda}+X^{*}\right)\left(3\left(\beta^{*}+1\right) \lambda^{2}-2 \beta^{*} \lambda+X^{*}\right)}
\end{gathered}
$$

where $X^{*}=\beta^{*}-A+1$. It follows that

$$
\left.\frac{d|\lambda|^{2}}{d \beta}\right|_{\beta=\beta^{*}}=-\frac{(A-2)(A+2)(A+1)}{\alpha_{1}^{2}+\alpha_{2}^{2}}
$$

where

$$
\begin{aligned}
& \alpha_{1}=3\left(\beta^{*}+1\right) \cos \left(2 \theta^{*}\right)-2 \beta^{*} \cos \theta^{*}+\left(\beta^{*}-A+1\right) \\
& \alpha_{2}=3\left(\beta^{*}+1\right) \sin \left(2 \theta^{*}\right)-2 \beta^{*} \sin \theta^{*}
\end{aligned}
$$

We conclude that $\left.\frac{d|\lambda|^{2}}{d \beta}\right|_{\beta=\beta^{*}} \neq 0$ for $A \in(0,1)$ which is the required result.

This completes also the proof of theorem (3.1).

\section{Direction of Neimark-Sacker bifurcation}

We have shown that system (2.1) undergoes a Neimark-Sacker bifurcation. In this section, we determine the direction of stability of the invariant closed curve bifurcating from the positive fixed point. We follow the the normal form theory of Neimark-Sacker bifurcation as in Kuznetsove, [5], see also [2].

Now, we shift the fixed point to the origin by taking $u_{n}=x_{n}-x^{*}, v_{n}=y_{n}-y^{*}, w_{n}=z_{n}-z^{*}$. System (2.1) takes the form

$$
\begin{aligned}
u_{n+1} & =\frac{B\left(u_{n}+X^{*}\right)+w_{n}+X^{*}}{A+v_{n}+X^{*}}-X^{*} \\
v_{n+1} & =u_{n} \\
w_{n+1} & =v_{n}
\end{aligned}
$$

Which can be written as

$$
Y_{n+1}=J Y_{n}+G\left(Y_{n}\right)+O\left(\|Y\|^{4}\right)
$$

where

$$
G(Y)=\frac{1}{2} B(Y, Y)+\frac{1}{6} C(Y, Y, Y), \quad \text { and } \quad Y_{n}=\left(u_{n}, v_{n}, w_{n}\right)^{T} \in \mathbb{R}^{3}
$$

and

$$
B(Y, Y)=\left(B_{1}(Y, Y), 0,0\right)^{T} \quad \text { and } \quad C(Y, Y, Y)=\left(C_{1}(Y, Y, Y), 0,0\right)^{T}
$$

where

$$
B_{i}(\xi, \zeta)=\left.\sum_{j, k=1}^{n} \frac{\partial^{2} Y_{i}(\phi)}{\partial \phi_{j} \partial \phi_{k}}\right|_{\phi=0} \xi_{j} \zeta_{k}
$$

and

$$
C_{i}(\xi, \zeta, \eta)=\left.\sum_{j, k, l=1}^{n} \frac{\partial^{3} Y_{i}(\phi)}{\partial \phi_{j} \partial \phi_{k} \partial \phi_{l}}\right|_{\phi=0} \xi_{j} \zeta_{k} \eta_{l}
$$




$$
\begin{gathered}
B_{1}(\xi, \zeta)=\frac{-\beta}{(\beta+1)^{2}}\left(\xi_{2} \zeta_{1}+\xi_{1} \zeta_{2}\right)+\frac{2(\beta-A+1)}{(\beta+1)^{2}} \xi_{2} \zeta_{2}-\frac{1}{(\beta+1)^{2}}\left(\xi_{3} \zeta_{2}+\xi_{2} \zeta_{3}\right) \\
C_{1}(\xi, \zeta, \eta)=\frac{2}{(\beta+1)^{3}}\left(\xi_{1} \zeta_{2} \eta_{2}+\xi_{2} \eta_{1} \zeta_{2}+\xi_{2} \zeta_{2} \eta_{1}\right) \\
+\frac{2 \beta}{(\beta+1)^{3}}\left(\xi_{2} \zeta_{2} \eta_{3}+\xi_{2} \zeta_{3} \eta_{2}+\xi_{3} \zeta_{2} \eta_{2}\right)-\frac{6(\beta-A+1)}{(\beta+1)^{3}} \xi_{2} \zeta_{2} \eta_{2}
\end{gathered}
$$

Let $q^{*} \in \mathbb{C}^{3}$ be an eigenvector of $J$ corresponding to the eigenvalue $e^{i \theta^{*}}$ and $p^{*} \in \mathbb{C}^{3}$ be an eigenvector of $J^{T}$ corresponding to the eigenvalue $e^{-i \theta^{*}}$; that is,

$$
J q^{*}=e^{i \theta^{*}} q^{*}, J^{T} p^{*}=e^{-i \theta^{*}} p^{*}
$$

Solving $(J-\lambda I) q^{*}=\left(J-e^{i \theta} I\right) q^{*}=0$, we get $q^{*} \sim\left(1, e^{-i \theta^{*}}, e^{-2 i \theta^{*}}\right)^{T}$ and solving $(J-\lambda I)^{T} p^{*}=\left(J-e^{-i \theta^{*}} I\right)^{T} p^{*}=0$ we get $p^{*} \sim$ $\left(1, e^{-i \theta^{*}}-\frac{\beta}{\beta+1}, \frac{e^{i \theta^{*}}}{\beta+1}\right)^{T}$. Now, we want to normalize $p^{*}$ and $q^{*}$ so that $\left\langle q^{*}, p^{*}\right\rangle=1$, where $\langle.,$.$\rangle is the standard scalar product in \mathbb{C}^{3}$. Note that

$$
\left\langle q^{*}, p^{*}\right\rangle=\sum_{i=1}^{3} \overline{q_{i}} p_{i}=2-\frac{\beta e^{i \theta^{*}}}{\beta+1}+\frac{e^{3 i \theta^{*}}}{\beta+1}
$$

So let $q=\varphi q^{*}$ where $\varphi=\left(2-\frac{\beta e^{i \theta^{*}}}{\beta+1}+\frac{e^{3 i \theta^{*}}}{\beta+1}\right)^{-1}$ and $p=p^{*}$. The real eigenspace $T^{c}$ corresponding to $\lambda_{1,2}$ is two-dimensional and is spanned by $\{\operatorname{Re}(q), \operatorname{Im}(q)\}$. The real eigenspace $T^{s}$ corresponding to the real eigenvalue of $J$ is one-dimensional. Any vector $x \in \mathbb{R}^{3}$ may be decomposed as

$$
x=z q+\bar{z} \bar{q}+y
$$

where $z \in \mathbb{C}^{1}$, and $\bar{z} \bar{q} \in T^{c}, y \in T^{s u}$. The complex variable $z$ is a coordinate on $T^{c}$. We have

$$
\begin{aligned}
z & =\langle p, x\rangle \\
y & =x-\langle p, x\rangle q-\langle\bar{p}, x\rangle \bar{q}
\end{aligned}
$$

In these coordinates, the map (4.1) takes the form

$$
\begin{aligned}
z & \mapsto e^{i \theta^{*}} z+\langle p, G(z q+\bar{z} \bar{q}+y)\rangle \\
y & \mapsto J y+G(z q+\bar{z} \bar{q}+y)-\langle p, G(z q+\bar{z} \bar{q}+y)\rangle q-\langle\bar{p}, G(z q+\bar{z} \bar{q}+y)\rangle \bar{q}
\end{aligned}
$$

Using Taylor expansions, the previous system can be written in the form:

$$
\begin{aligned}
z & \mapsto \quad e^{i \theta^{*}} z+\frac{1}{2} G_{20} z^{2}+G_{11} z \bar{z}+\frac{1}{2} G_{02} \bar{z}^{2}+\frac{1}{2} G_{21} z^{2} \bar{z}+\cdots \\
y & \mapsto J y+\frac{1}{2} H_{20} z^{2}+H_{11} z \bar{z}+\frac{1}{2} H_{02} \bar{z}^{2}+\cdots
\end{aligned}
$$

Where

$$
G_{20}=\langle p, B(q, q)\rangle, G_{11}=\langle p, B(q, \bar{q})\rangle, G_{02}=\langle p, B(\bar{q}, \bar{q})\rangle
$$

and

$$
\begin{gathered}
G_{21}=\langle p, C(q, q, \bar{q})\rangle \\
H_{20}=B(q, q)-\langle p, B(q, q)\rangle q-\langle\bar{p}, B(q, q)\rangle \bar{q} \\
H_{11}=B(q, \bar{q})-\langle p, B(q, \bar{q})\rangle q-\langle\bar{p}, B(q, \bar{q})\rangle \bar{q}
\end{gathered}
$$

and the scalar product in $\mathbb{C}^{3}$ is used. From the center manifold theorem, there exists a center manifold $W^{c}$ which can be approximated as

$$
Y=V(z, \bar{z})=\frac{1}{2} w_{20} z^{2}+w_{11} z \bar{z}+\frac{1}{2} w_{02} \bar{z}^{2}+O\left(|z|^{3}\right)
$$

where $\left\langle p, w_{i j}\right\rangle=0$. The vectors $w_{i j} \in \mathbb{C}^{3}$ can be found from the linear equations

$$
\begin{aligned}
& w_{20}=\left(e^{2 i \theta^{*}} I_{3}-J\right)^{-1} H_{20} \\
& w_{11}=\left(I_{3}-J\right)^{-1} H_{11} \\
& w_{02}=\left(e^{-2 i \theta^{*}} I_{3}-J\right)^{-1} H_{02}
\end{aligned}
$$

So $z$ can be expressed as

$$
z \mapsto e^{i \theta^{*}} z+\frac{1}{2} G_{20} z^{2}+G_{11} z \bar{z}+\frac{1}{2} G_{02} \bar{z}^{2}+\frac{1}{2}\left(G_{21}+2\left\langle p, B\left(q,(I-J)^{-1} H_{11}\right)\right\rangle\right.
$$




$$
\left.+\left\langle p, B\left(\bar{q},\left(e^{2 i \theta^{*}} I-J\right)^{-1} H_{20}\right)\right\rangle\right) z^{2} \bar{z}
$$

Substituting equations (4.2)-(4.5) into (4.6) and taking into account the identities

$$
(I-J)^{-1} q=\frac{1}{1-e^{i \theta^{*}}} q, \quad\left(e^{2 i \theta^{*}} I-J\right)^{-1} q=\frac{e^{-i \theta^{*}}}{e^{i \theta^{*}}-1} q
$$

and

$$
(I-J)^{-1} \bar{q}=\frac{1}{1-e^{-i \theta^{*}}} \bar{q}, \quad\left(e^{2 i \theta^{*}} I-J\right)^{-1} \bar{q}=\frac{e^{i \theta^{*}}}{e^{3 i \theta^{*}}-1} \bar{q}
$$

We can express $z$ using the map

$$
z \mapsto e^{i \theta^{*}} z+\sum_{k+l \geq 2} \frac{1}{k ! j !} g_{k j} z^{k} \bar{z}^{j}
$$

Finally, the restricted map can be written as

$$
z \mapsto e^{i \theta^{*}} z\left(1+d\left(\beta^{*}\right)\right)|z|^{2}+O\left(|z|^{4}\right)
$$

where the real number $A\left(\beta^{*}\right)=\operatorname{Re}\left(d\left(\beta^{*}\right)\right)$ determines the direction of bifurcation of the closed invariant curve and can be computed using the formula

$$
A\left(\beta^{*}\right)=\operatorname{Re}\left(\frac{e^{-i \theta^{*}} g_{21}}{2}\right)-\operatorname{Re}\left(\frac{\left(1-2 e^{i \theta^{*}}\right) e^{-2 i \theta^{*}}}{2\left(1-e^{i \theta^{*}}\right)} g_{20} g_{11}\right)-\frac{1}{2}\left|g_{11}\right|^{2}-\frac{1}{4}\left|g_{02}\right|^{2}
$$

The coefficients $g_{20}, g_{11}, g_{02}$ and $g_{21}$ can be readily calculated using simple, but tedious, calculations. Firstly, we have

$$
B(q, q)=\left(\begin{array}{c}
\frac{2(\beta-A+1) e^{-2 i \theta^{*}}-2 \beta e^{-i \theta^{*}}-2 e^{-3 i \theta^{*}}}{(\beta+1)^{2}} \\
0 \\
0
\end{array}\right)
$$

It follows that

$$
g_{20}=\langle p, B(q, q)\rangle=\frac{2(\beta-A+1) e^{-2 i \theta^{*}}-2 \beta e^{-i \theta^{*}}-2 e^{-3 i \theta^{*}}}{(\beta+1)\left(2(\beta+1)-\beta e^{i \theta^{*}}+e^{3 i \theta^{*}}\right)}
$$

whereas

$$
B(q, \bar{q})=\left(\begin{array}{c}
\frac{2(\beta-A+1)-2(\beta+1) \cos \theta^{*}}{(\beta+1)^{2}} \\
0 \\
0
\end{array}\right)
$$

Hence,

$$
g_{11}=\langle p, B(q, \bar{q})\rangle=\frac{2(\beta-A+1)-2(\beta+1) \cos \theta^{*}}{(\beta+1)\left(2(\beta+1)-\beta e^{i \theta^{*}}+e^{3 i \theta^{*}}\right)}
$$

and

$$
B(\bar{q}, \bar{q})=\left(\begin{array}{c}
\frac{2(\beta-A+1) e^{2 i \theta^{*}}-2 \beta e^{i \theta^{*}}-2 e^{3 i \theta^{*}}}{(\beta+1)^{2}} \\
0 \\
0
\end{array}\right)
$$

Then

$$
g_{02}=\langle p, B(\bar{q}, \bar{q})\rangle=\frac{2(\beta-A+1) e^{2 i \theta^{*}}-2 \beta e^{i \theta^{*}}-2 e^{3 i \theta^{*}}}{(\beta+1)\left(2(\beta+1)-\beta e^{i \theta^{*}}+e^{3 i \theta^{*}}\right)}
$$

Finally, to find $g_{21}$ we use the formula

$$
\begin{gathered}
g_{21}=\langle p, C(q, q, \bar{q})\rangle+2\left\langle p, B\left(q,(I-J)^{-1} B(q, \bar{q})\right)\right\rangle+ \\
\left\langle p, B\left(\bar{q},\left(e^{2 i \theta^{*}} I-J\right)^{-1} B(q, q)\right)\right\rangle+\frac{e^{-i \theta^{*}}\left(1-2 e^{i \theta^{*}}\right)}{1-e^{i \theta^{*}}}\langle p, B(q, q)\rangle\langle p, B(q, \bar{q})\rangle \\
-\frac{2}{1-e^{-i \theta^{*}}}|\langle p, B(q, \bar{q})\rangle|^{2}-\frac{e^{i \theta^{*}}}{e^{3 i \theta^{*}}-1}|\langle p, B(\bar{q}, \bar{q})\rangle|^{2}
\end{gathered}
$$

where

$$
C(q, q, \bar{q})=\left(\begin{array}{c}
\frac{-6(B-A+1) e^{-i \theta^{*}}+2 B\left(1+2 e^{-2 i \theta^{*}}\right)+2\left(2+e^{-2 i \theta^{*}}\right)}{(B+1)^{3}} \\
0 \\
0
\end{array}\right)
$$


So

$$
\langle p, C(q, q, \bar{q})\rangle=\frac{-6(\beta-A+1) e^{-i \theta^{*}}+2 \beta\left(1+2 e^{-2 i \theta^{*}}\right)+2\left(2+e^{-2 i \theta^{*}}\right)}{(\beta+1)^{2}\left(2(\beta+1)-\beta e^{i \theta^{*}}+e^{3 i \theta^{*}}\right)}
$$

and

$$
\left\langle p, B\left(\bar{q},\left(e^{2 i \theta^{*}} I-J\right)^{-1} B(q, q)\right\rangle=\frac{L\left(2(\beta-A+1) e^{3 i \theta^{*}}-\beta\left(e^{2 i \theta^{*}}+e^{5 i \theta^{*}}\right)-\left(e^{i \theta^{*}}+e^{4 i \theta^{*}}\right)\right)}{K\left(2(\beta+1)-\beta e^{i \theta^{*}}+e^{3 i \theta^{*}}\right)}\right.
$$

where

$$
K=(\beta+1) e^{6 i \theta_{9}}-\beta e^{4 i \theta^{*}}+(\beta-A+1) e^{2 i \theta^{*}}-1, L=\frac{2(\beta-A+1)-2(\beta+1) \cos \theta^{*}}{(\beta+1)^{2}}
$$

Depending on the above calculation, we find that $A\left(\beta^{*}\right)=-0.91<0$ when $A=0.5, \beta=\beta^{*}=1 / 3$, so the closed invariant curve is supercritical (stable) according to the following theorem.

Theorem 4.1. If $A\left(\beta^{*}\right)<0$ (respectively, $>0$ ), then the Neimark-Sacker bifurcation at $\beta=\beta^{*}$ is supercritical (respectively, subcritical) and there exists a unique invariant closed curve bifurcates from the fixed point which is asymptotically stable (respectively, unstable).

\section{Computer simulation}

In this section, we present some numerical simulations of equation (1.2) that supports our analytical results. The first figure is a bifurcation diagram for equation (1.2) when $A=0.5, x_{-2}=x_{-1}=x_{0}=0.2$. In this case, the positive equilibrium point is stable if $\beta>\frac{1}{3}$ and unstable if $\beta<\frac{1}{3}$. In figures 2 and 3 , we plot phase portraits in the $(x(n), x(n-2))$ plane. In Figure $2, A=0.5, \beta=\beta^{*}$, and $x_{-2}=x_{-1}=x_{0}=0.2$. Notice the existence of a closed invariant curve at the bifurcation value. In figure $3, A=0.5, \beta=0.4$, and $x_{-2}=x_{-1}=x_{0}=0.5$.

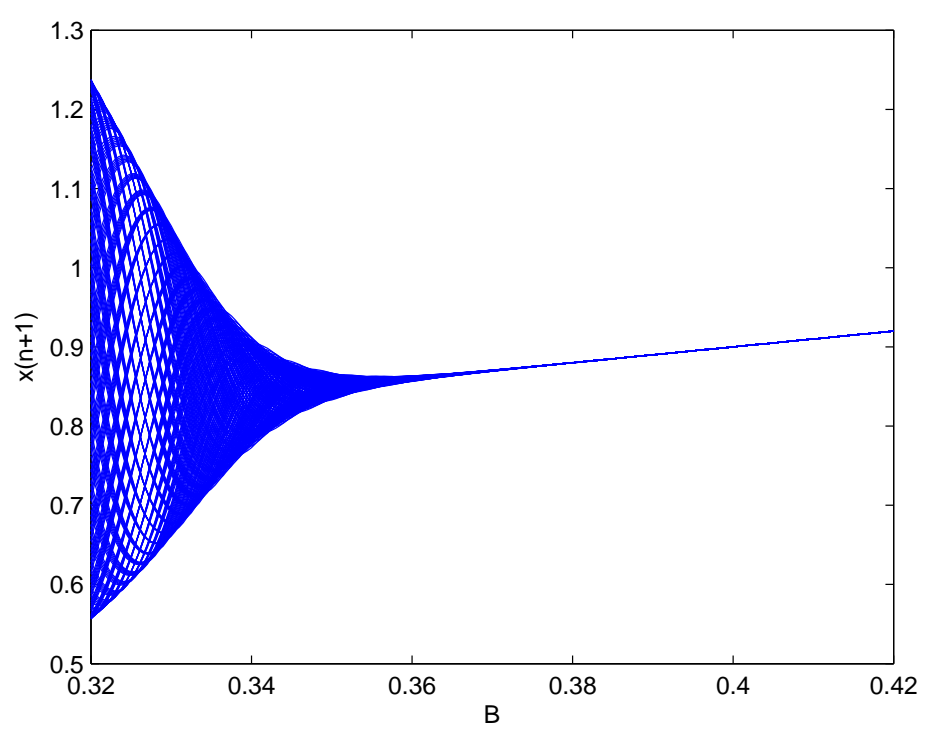

Figure 1: Bifurcation diagram of Eq.(1.2) in $(\beta, X)$ plane for $A=0.5$ 


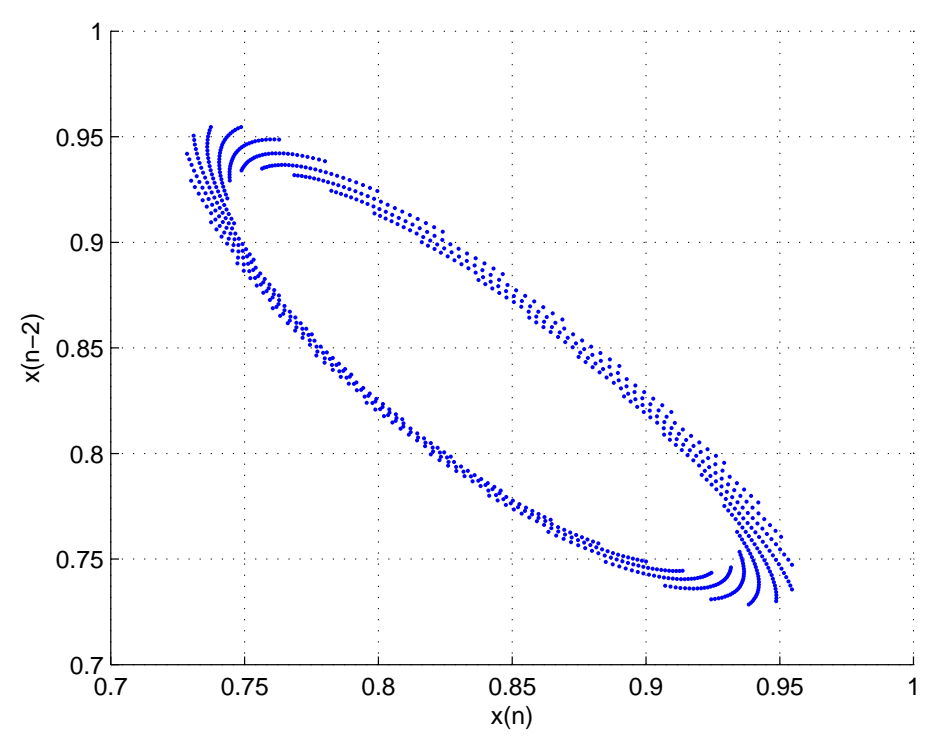

Figure 2: Phase portrait of Eq.(1.2) in $(x(n), x(n-2))$ plane for $A=0.5, \beta=1 / 3$

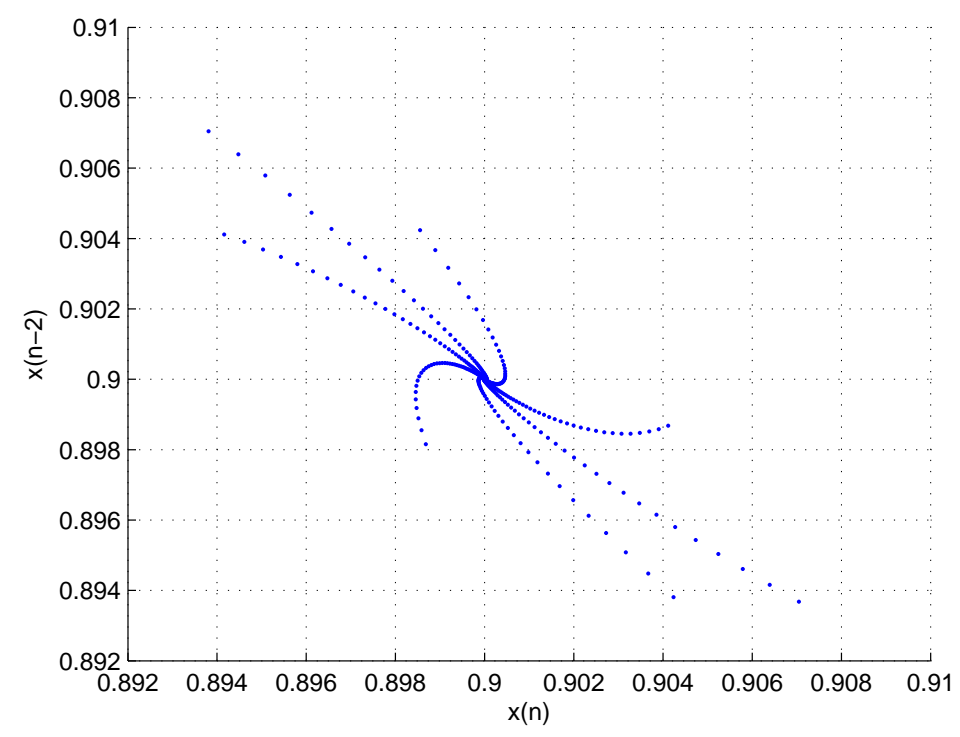

Figure 3: Phase portrait of Eq.(1.2) in $(x(n), x(n-2))$ plane for $A=0.5, \beta=0.4$

\section{Conclusion}

In this paper, we have used normal form theory to show that a third order difference equation undergoes a Neimar-Sacker bifurcation. All conditions for the existence of A Neimark-Sacker bifurcation have been checked. In the last section, we gave some numerical simulations that support our analytical results. Notice the stability of the invariant curve and the fixed point in figure 2 and figure 3 , respectively, as predicted by Theorem 4.1 .

\section{References}

[1] E. Camouzis, Global analysis of solutions of $x_{n+1}=\frac{\beta x_{n}+\delta x_{n-2}}{A+B x_{n}+C x_{n-1}}$, J. Math Anal. Appl., 316 (2005), 616-627.

[2] Z. He, J. Qiu, Neimark-Sacker bifurcation of a third order rational difference equation, J. Differ. Equ. Appl.,19 (2013), $1513-1522$.

[3] E. Camouzis, G. Ladas, Dynamics of Third-Order Rational Difference Equations with Open Problems and Conjectures, Chapman \& Hall/CRC, New York, 2002

[4] A. D. Polyanin, A. I. Chernoutsan, A. Concise, A Concise Handbook of Mathematics, Physics and Engineering Science, CRC Press, New York, 2011.

[5] Y. Kuznetsov, Elements of Applied Bifurcation Theory, 2nd edition, Springer, New York, 2003. 